\title{
Delineating the hierarchy of lung progenitor cells and their response to influenza
}

\author{
Jonathan L. McQualter ${ }^{1}$ and Geoffrey J. Laurent ${ }^{2,3}$ \\ Affiliations: ${ }^{1}$ RMIT University, School of Health Sciences, Melbourne, Australia. ${ }^{2}$ Centre for Cell Therapy and \\ Regenerative Medicine, School of Medicine and Pharmacology, The University of Western Australia, Perth, \\ Australia. ${ }^{3}$ Institute for Respiratory Health, Harry Perkins Institute of Medical Research, Perth, Australia.
}

Correspondence: Jonathan L. McQualter, RMIT University, School of Health Sciences, P.O. Box 71, Bundoora, VIC 3083, Australia. E-mail: jonathan.mcqualterdrmit.edu.au

$\circ$ @ERSpublications

Regeneration of mouse alveoli after influenza infection involves rare stem cells in the bronchiolar airways http://ow.ly/NUB7P

Influenza viruses are significant human respiratory pathogens that cause acute inflammation and widespread epithelial cell death in the lungs [1]. Yet, patients can recover, even after widespread destruction of epithelial cells, implying that the lung has a tremendous capacity for regeneration after injury. The mechanism by which this wound-healing process occurs remains unclear. In the adult mouse lung, current models of lung repair have established that different cell types participate in the wound-healing response depending on the nature, anatomical region of the lung (tracheobronchial airways, bronchiolar airways or the alveoli) and extent of the injury. Basal cells are the main stem cell population in the tracheobronchial airways, club cells serve as abundant progenitors in the bronchiolar airways, and type II cells support the maintenance of the alveoli. There is also convincing evidence for the existence of a multipotent stem cell population(s) that can give rise to both airway and alveolar lineages, but the precise identity of these stem cells and their response to lung injury remains unclear $[2,3]$. Thus, a key question remains: are rare stem cell populations aroused from their quiescent state to participate in de novo tissue regeneration after extensive epithelial injury?

Influenza virus infection in mice causes widespread ablation of epithelial cells with discrete clusters of cytokeratin 5-positive $\left(\mathrm{Krt}^{+}\right)$cells appearing in the alveolar gas exchange region [4]. The emergence of these induced $\mathrm{Krt}^{+}$cells hinted that this might represent the early stages of de novo alveoli formation. However, the cellular origin of the induced $\mathrm{Krt}^{+}$cells is unclear and evidence for their definite integration into regenerated alveolar tissue has been lacking [4]. In an exciting new study reported in Nature, VAUGHAN et al. [5] identified a rare population of stem cells that is activated after influenza virus infection. Using multiple fate mapping strategies, the researchers showed that after activation, these stem cells contribute substantially to the induced $\mathrm{Krt}^{+}$cells that emerge after influenza virus infection in mice. They also reported that induced $\mathrm{Krt}^{+}$cells also appear after bleomycin injury, albeit to a lesser extent than after influenza virus infection. This suggests that the $\mathrm{Krt}^{+}{ }^{+}$regeneration programme is not a specific response to the influenza virus infection, but rather is engaged after different forms of epithelial injury. Still, it is unclear whether induction of these cells is part of the normal wound-healing response or whether this represents an emergency regenerative response in the case of severe injury. Certainly, several studies have shown that club cell-specific protein (CC10) and surfactant protein C (SPC) lineage-tagged cells contribute to alveolar regeneration (reviewed in [2]). Further studies with diverse injury models should now be undertaken to determine the circumstance in which the $\mathrm{Krt}^{+}$regenerative programme is activated, to determine its relevance for human diseases such as acute respiratory distress syndrome, chronic obstructive pulmonary disease (COPD) and pulmonary fibrosis.

To establish lineage identity of induced $\mathrm{Krt5}^{+}$cells, VAUGHAN et al. [5] used transgenic mice with lineage-specific, tamoxifen-dependent Cre recombinase activity to indelibly tag cells of interest and all of

Received: March 012015 | Accepted after revision: April 112015

Conflict of interest: None declared.

Copyright @ERS 2015 
their progeny with fluorescent reporters. They reported that lineage-tagged $\mathrm{Krt} 5^{+}$cells contributed to only $13 \%$ of the induced $\mathrm{Krt}^{+}$cells after influenza virus infection. Although this may be an underestimate depending on the efficiency of the tamoxifen-induced recombination of the reporter gene, it suggests that existing $\mathrm{Krt5}^{+}$cells are not the cellular origin for the majority of the induced $\mathrm{Krt5}^{+}$cells. In a parallel study, reported in the same issue of Nature, Zuo et al. [6] showed that over $50 \%$ of induced $\mathrm{Krt}^{+}$cells were derived from existing lineage-tagged $\mathrm{Krt}^{+}$cells, which they dubbed distal airway stem cells (DASCs). The differences between these two studies may be due to the fact that Zuo et al. [6] used a transgene driven by the bovine Krt5 promoter, whereas VAUGHAN et al. [5] used a transgene that was inserted into the 3' untranslated region of the endogenous Krt5 gene. Nevertheless, both reports raise the possibility that existing $\mathrm{Krt}^{+}$basal cells in the proximal airways might migrate distally after alveolar injury. VAUGHAN et al. [5] tested this scenario by transplanting sections of fluorescent trachea into syngeneic recipients and looking for the migration of fluorescently tagged cells after influenza virus infection. They showed that none of the induced $\mathrm{Krt}^{+}$cells that arose were fluorescently tagged, suggesting that it is unlikely that tracheal basal cells contribute to the population of induced $\mathrm{Krt}^{+}$cells. However, one should consider the possibility that the transplantation procedure may impede the migration of cells, and that some of the $\mathrm{Krt} 5^{+}$basal cells residing in the remaining host airways may contribute to the population of induced $\mathrm{Krt}^{+}$cells.

VAUGHAN et al. [5] also dismissed a role for existing $\mathrm{SPC}^{+}$and $\mathrm{CC}_{10}{ }^{+}$cells in the formation of induced $\mathrm{Krt}^{+}$cell clusters, reporting that none of the induced $\mathrm{Krt}^{+}$cells were lineage tagged using SPC-CreERT2 and CC10-CreERT2 reporters. Hence, they proposed a lineage-negative epithelial progenitor (LNEP) as the major source of induced $\mathrm{Krt5}^{+}$cells. Interestingly, however, they did show that for CC10-CreERT2 reporters this was dependent on the chase time between tamoxifen injection and influenza virus infection. With a shorter chase time ( 7 days versus 21 days), about $40 \%$ of the induced $\mathrm{Krt}^{+}$cells were lineage tagged. This is consistent with a previous study showing that existing $\mathrm{CC} 10^{+}$cells contributed significantly to the induced $\mathrm{Krt5}^{+}$cells [7]. VAughan et al. [5] attribute this to tamoxifen persistence, showing that Krt5-CreERT2-tagged cells expressed detectable levels of CC10 by day 11 and onwards post influenza virus infection. While this is a plausible explanation, the turnover of induced $\mathrm{Krt5}^{+}$cells is unknown. Thus, it is worth considering the possibility that CC10-CreERT2-tagged cells do contribute to the population of induced $\mathrm{Krt}^{+}$cells, but the expression of $\mathrm{Krt5}^{+}$is short-lived before cells further differentiate. Unfortunately, the authors only reported data for 11 days post infection and the expression of other differentiation markers in CC10-CreERT2-tagged cells was not assessed.

In a major technological advance, VAUGHAN et al. [5] developed an orthotopic transplantation model to demonstrate the proliferative capacity and multilineage specificity of the putative progenitor cells. This allowed them to use fluorescence-activated cell sorter segregation of epithelial cells, based on surface markers identified from single-cell transcriptome analysis, to isolate putative LNEPs with transplant potential. They demonstrated that putative LNEPs, enriched in the EpCAM ${ }^{+} \operatorname{Itg} \beta 4^{+} \mathrm{CD} 200^{+} \mathrm{CD} 14^{+}$cell fraction, were readily transplantable and exhibited the capacity for multilineage differentiation into $\mathrm{CC}_{10}{ }^{+}$, $\mathrm{Krt}^{+}$or $\mathrm{SPC}^{+}$. Interestingly, they reported that Krt5-CreERT2-tagged cells within the putative LNEP fraction were also readily transplantable, but gave rise to fewer $\mathrm{SPC}^{+}$cells, indicating heterogeneity in the lineage specificity of these cells. Intriguingly, the LNEPs were also shown to express low levels of the CC10 and SPC genes, albeit significantly lower than the respective differentiated cells, which raises the question of appropriate use of nomenclature. Where do you draw the line to differentiate between lineage-negative and low-expressing cells?

VAUGHAN et al. [5] also revealed that the putative LNEPs uniquely expressed $\Delta$ Np63. In uninjured lungs, $\Delta \mathrm{Np} 3^{+}$cells could be observed scattered sporadically throughout the distal airways, but only a fraction of these cells were Krt5-CreERT2 tagged. Thus, VAughan et al. [5] proposed that rare LNEPs residing in the bronchiolar airways are activated after influenza virus infection to expand and give rise to induced $\mathrm{Krt}-5^{+}$ cells in the alveolar parenchyma and more differentiated lineage-committed bronchiolar and alveolar epithelial cells (figure 1). Notably, the researchers used live-cell imaging of lung slices to demonstrate that Krt5-CreERT2-tagged cells were able to migrate through the lung parenchyma after influenza virus infection. Future studies should interrogate the mechanisms driving migration. What are the factors driving migration of progenitors to areas of epithelial ablation after influenza virus infection? Is there a requirement for remodelling of the extracellular matrix for migration to proceed? How do the inflammatory cells and stromal cells that are closely associated with induced $\mathrm{Krt5}^{+}$cell clusters influence the fate and specificity of LNEPs?

It remains unclear how heterogeneous the lung stem cell population is, and whether any specific stem cell population is essential for the regenerative response. In an attempt to address this, Zuo et al. [6] showed that conditional ablation of $\mathrm{Krt}^{+}$cells hampered tissue regeneration after influenza virus infection. The researchers used transgenic mice expressing the diphtheria toxin receptor driven by the cytokeratin 6 (Krt6) promoter, rather than the more broadly expressed Krt5 promoter. They previously reported that Krt6 was uniquely expressed in DASC clones only after influenza virus infection. Thus, while this suggests 


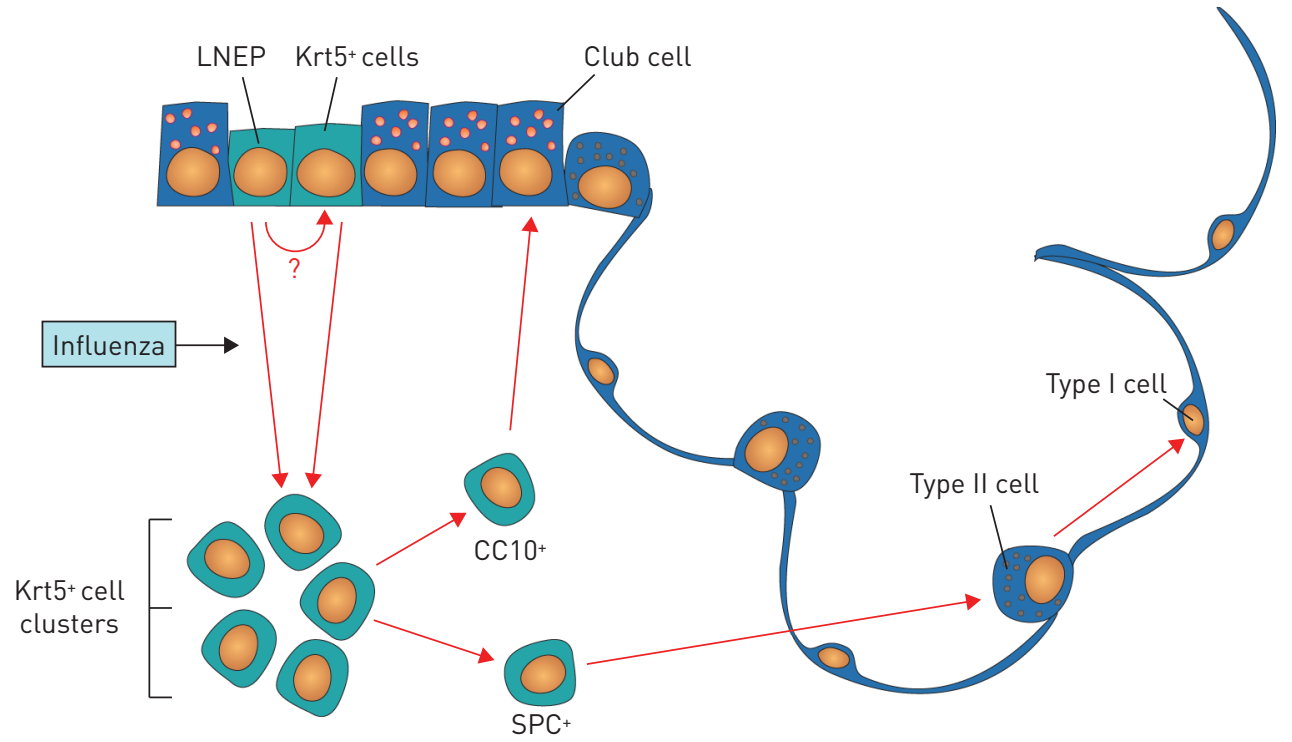

FIGURE 1 Schematic of lineage hierarchy of regenerative cells activated by influenza virus infection. VAUGHAN et al. [5] propose that rare lineage-negative epithelial progenitors (LNEPs) and/or cytokeratin 5-positive $\left(\mathrm{Krt}^{+}\right)$cells residing in the bronchiolar airways are activated after influenza virus infection to expand and migrate into the distal lung parenchyma, where they proliferate into $\mathrm{Krt5}^{+}$cell clusters and then differentiate into cells bearing markers of alveolar type II and type I cells or bronchiolar club cells. CC10: club cell-specific protein; SPC: surfactant protein C.

that the induced $\mathrm{Krt5}^{+} \mathrm{Krt6}^{+}$cells are directly involved in the regenerative process after influenza virus infection, it offers no further clues to their cell of origin.

Some insight into the mechanisms directing the fate of putative LNEPs was provided by the demonstration that Notch signalling was required for the expression of $\mathrm{Krt5}$, while its inhibition directed cells to differentiate into alveolar cells [5]. Furthermore, VAUGHAN et al. [5] identified areas of active Notch signalling in honeycomb cysts of lungs from patients with fibrosis. The researchers concluded that the outcomes of regeneration or fibrosis might depend on the dynamics of Notch signalling in LNEPs. Further studies should aim to clarify the molecular mechanisms directing cell fate decisions of epithelial progenitors that are activated after influenza virus infection or injury.

In conclusion, the two papers report that regeneration of the alveolar gas exchange region of the mouse lung after influenza virus infection involves the activation and migration of rare stem cells residing in the bronchiolar airways. However, it is far less clear whether there is a human correlate for LNEPs, DASCs or induced $\mathrm{Krt5}^{+}$cells. Clearly, the question of whether the Krt5 regeneration programme is relevant to human lung regeneration and whether it is altered in lung disease must be addressed in future studies. Further understanding of cellular and molecular mechanisms of the lung regeneration programme will provide exciting translational opportunities to stimulate the proliferation and differentiation of stem and progenitor cells to improve endogenous regeneration of the lung after injury. In addition to its obvious relevance to the many human deaths attributed to influenza each year, this research provides exciting translational opportunities for new treatment options for patients with chronic lung disease such as COPD and pulmonary fibrosis, which may reflect a regenerative process gone awry.

\section{References}

1 Taubenberger JK, Morens DM. The pathology of influenza virus infections. Annu Rev Pathol 2008; 3: 499-522.

2 McQualter JL, Anthony D, Bozinovski S, et al. Harnessing the potential of lung stem cells for regenerative medicine. Int J Biochem Cell Biol 2014; 56: 82-91.

3 Hogan BL, Barkauskas CE, Chapman HA, et al. Repair and regeneration of the respiratory system: complexity, plasticity, and mechanisms of lung stem cell function. Cell Stem Cell 2014; 15: 123-138.

4 Kumar PA, Hu Y, Yamamoto Y, et al. Distal airway stem cells yield alveoli in vitro and during lung regeneration following H1N1 influenza infection. Cell 2011; 147: 525-538.

5 Vaughan AE, Brumwell AN, Xi Y, et al. Lineage-negative progenitors mobilize to regenerate lung epithelium after major injury. Nature 2015; 517: 621-625.

6 Zuo W, Zhang T, Wu DZ, et al. $\mathrm{p}^{+} 3^{+} \mathrm{Krt}^{+}$distal airway stem cells are essential for lung regeneration. Nature 2015; 517: 616-620.

7 Zheng D, Yin L, Chen J. Evidence for Scgbla1 ${ }^{+}$cells in the generation of $\mathrm{p}^{+} 3^{+}$cells in the damaged lung parenchyma. Am J Respir Cell Mol Biol 2014; 50: 595-604. 